\title{
An Algorithm to Detect Packet Forwarding Misbehavior in Mobile Ad-Hoc Networks
}

\author{
Oscar F. Gonzalez, Michael Howarth, George Pavlou \\ Center for Communications Systems Research \\ University of Surrey \\ Guildford, UK \\ O.Gonzalez-Duque@surrey.ac.uk, M.Howarth@surrey.ac.uk, G.Pavlou@surrey.ac.uk
}

\begin{abstract}
Devices in a Mobile Ad Hoc network (MANET) may misbehave by dropping packets that are forwarded in the network, for example because they are malicious and deliberately intend to disrupt the network, or because they are selfish and wish to conserve their own limited resources such as power. In this paper, we present a mechanism that enables the detection of nodes that exhibit packet forwarding misbehavior. We present initial evaluation results that demonstrate the operation of our algorithm and show that it effectively detects nodes that drop a significant fraction of packets.
\end{abstract}

Keywords-mobile ad hoc network, misbehavior detection, packet forwarding.

\section{INTRODUCTION}

The wireless nature and inherent features of mobile ad hoc networks make them vulnerable to a wide variety of attacks by misbehaving nodes. Such attacks range from passive eavesdropping, where a node tries to obtain unauthorized access to data destined for another node, to active interference where malicious nodes hinder network performance by not obeying globally acceptable rules. Misbehavior can be divided into two categories [1]: routing misbehavior (failure to behave in accordance with a routing protocol) and packet forwarding misbehavior (failure to correctly forward data packets in accordance with a data transfer protocol). In this paper we focus on the latter type of misbehavior. Our approach consists of an algorithm that enables packet forwarding misbehavior detection through the principle of flow conservation [3]. Our scheme is not tightly coupled to any specific routing protocol and, therefore, it can operate regardless of the routing strategy adopted. Our criterion for judging a node is the estimated percentage of packets dropped, which is compared against a pre-established misbehavior threshold. Any node dropping packets in excess of this threshold is deemed a misbehaving node while those below the threshold are considered to be correctly behaving.

In this paper we first present a framework and an algorithm and protocol that deal with this attack. We then demonstrate through simulations that an appropriate selection of the misbehavior threshold allows for a good discrimination between misbehaved and well-behaved nodes in a network that is affected by black hole attacks, where malicious nodes drop all packets they receive, and/or gray hole attacks, where they drop packets selectively or in a probabilistic manner in an attempt to circumvent the security measures in place.

Section $I$ of this paper describes our algorithm for packet forwarding misbehavior detection, and Section III presents an initial performance evaluation. Finally, the paper is summarized in Section IV.

\section{DETECTING PACKET FoRW ARDING MiSBEHAVIOR}

Our work provides a novel approach to securing the data forwarding functionality in mobile ad hoc networks. We propose an approach that takes advantage of the principle of flow conservation in a network. This states that all bytes/packets sent to a node, and not destined for that node, are expected to exit the node. In this Section we first present, from a theoretical point of view, how this principle works assum ing it is implemented in an ideal network, and then we demonstrate that by making some reasonable assumptions and adaptations, our algorithm can cope with the practical problems that are encountered in real MANETs.

\section{A. The Principle of Flow Conservation}

We formally introduce the principle of flow conservation over an ideal static network model:

- Let $v_{j}$ be a node such that $v_{j} \in V$, where $V=\left\{v_{l}, v_{2}, v_{3} \ldots\right.$ $v_{N j}$ is the set of all nodes in the network, $N$ is the total number of nodes in the network, and $j=1,2,3 \ldots N$.

- Let $U_{j}$ be the subset of nodes in the network which are neighbors of $v_{j}$, i.e. $U_{j}$ is the neighborhood of $v_{j}$. It follows that $v_{j} \notin U_{j}$ and also $U_{j} \subset V$.

- Let $\Delta t$ be the period of time elapsed between two points in time $t_{0}$ and $t_{1}$ such that $\Delta t=t_{1}-t_{0}$.

- Let $T_{i j}$ be the number of packets that node $v_{i}$ has successfully sent to node $v_{j}$ for $v_{j}$ to forward to a further node; $v_{i} \in U_{j}, v_{j} \in U_{i}, i \neq j$ and $T_{i j}\left(t_{0}\right)=0$.

- Let $R_{i j}$ be the number of packets that node $v_{i}$ has successfully received from node $v_{j}$ that did not originate at $v_{j}, v_{i} \in U_{j}, v_{j} \in U_{i}, \quad i \neq j$ and $R_{i j}\left(t_{0}\right)=0$.

If all nodes $v_{j} \in V$ remain static for a period of time $\Delta t$ during which no collisions occur in any of the transmissions over an ideal (noiseless) wireless channel, then for a node $v_{j}$ : 
$\sum_{\forall i p_{i} \in U_{j}} R_{i j}\left(t_{1}\right)=\sum_{\forall i p_{i} \in U_{j}} T_{i j}\left(t_{1}\right)$

This equation states the fundamental premise of the flow conservation principle in an ideal static network, and is applied to packets rather than individual bytes. It states that if all neighbors of a node $v_{j}$ are queried for i) the amount of packets sent to $v_{j}$ to forward and ii) the amount of packets forwarded by $v_{j}$ to them, the total amount of packets sent to and received from $v_{j}$ must be equal.

In practice MANETs exhibit conditions that are far from ideal. First of all, the wireless channel is error prone and packets get lost while in transit. Secondly, collisions happen when the network uses protocols where nodes have to compete for the medium, such as when the link layer protocol is based on the distributed coordination function (contention period) of the IEEE $802.11 \mathrm{a} / \mathrm{b}$ standard. In order to allow equation (1) to hold our algorithm requires the use of a reliable MAC protocol such as IEEE 802.11, MACA [4] or MACAW [5], and the rest of this paper assumes that such a reliable MAC protocol is used.

A reliable MAC protocol at the link layer acknowledges each successfully transmitted packet and thus transmitter and receiver can maintain synchronized values of their metrics $T_{i j}$ and $R_{i j}$. The use of a reliable MAC protocol in conjunction with the conservation of flow principle means that we are not susceptible to problems that arise when overhearing other nodes' transmissions. Thus, problems such as ambiguous collisions, receiver collisions, and the ability of a node to control its transmission power, which affect the approaches proposed in [1] and [2], do not exist in our approach. Ambiguous collisions occur when a node $v_{l}$ is trying to determine if another node $v_{2}$ is properly forwarding a packet. It may happen that node $v_{2}$ forwards the packet to a further node $v_{3}$, which is out of the transmission range of $v_{l}$, while a second transmission prevents $v_{l}$ from overhearing the forwarded packet, thus $v_{l}$ will not know if the packet was forwarded. On the other hand, in the receiver collision problem $v_{2}$ forwards the packet to $v_{3}$ at which point a collision occurs. Node $v_{l}$ is unaware of such a collision and assumes that the packet was forwarded even if $v_{2}$ does not attempt a retransmission. Another common problem is caused by nodes capable of controlling their transmission power. Thus, $v_{2}$ can transmit with enough power for $v_{l}$ to overhear but not enough power for $v_{3}$ to receive it, leaving $v_{2}$ unaware of the situation. All these weaknesses, which can be used by malicious nodes to disrupt the network, are due to the fact that overhearing is used by nodes to check for misbehavior in other nodes. In our algorithm the nodes that maintain statistics that are used to determine whether the forwarding was properly made are the nodes actively involved in the transmission process, i.e. the transmitter and the receiver of each transmission.

However, a node may exhibit malicious behavior even if it is not purposefully doing so. For example, an overloaded node may temporarily lack the CPU cycles, buffer space or bandwidth to forward packets [2]. In addition, some reactive routing protocols, e.g. AODV, cause buffered packets to be dropped if they go through a path that is even temporarily unavailable. For these reasons equation (1) cannot be applied in a rigorous manner and a threshold needs to be established to account for packets dropped by a node through no fault of its own. Equation (2) reflects this change:

$$
\left(1-\alpha_{\text {threshold }}\right) \sum_{\forall i \mid v_{i} \in U_{j}} R_{i j}\left(t_{1}\right) \leq \sum_{\forall i \mid v_{i} \in U_{j}} T_{i j}\left(t_{1}\right)
$$

The $\alpha_{\text {threshold }}$ factor can take values between 0 and 1 and as we shall see plays an important role in the detection power of our proposed algorithm, i.e. the capability of the algorithm to detect misbehaving nodes. The lower $\alpha_{\text {threshold }}$ is the more likely it is that our algorithm detects any malicious behavior. However, it also means that the probability of a false detection increases. A false detection occurs when the result of a single evaluation of a node mistakenly determines that the node appears to be misbehaving. Therefore, fine tuning is required to reach a fair point in this tradeoff.

\section{B. Algorithm}

In MANETs the neighborhood $U_{j}$ of a node $v_{j}$ changes dynamically over time, making it difficult to determine those nodes that have transmitted or received packets to or from a node $v_{j}$. Our scheme overcomes this problem by means of a limited broadcast that tracks down nodes that have been in contact with node $v_{j \cdot}$. The core parts of our algorithm are detailed in the pseudocode shown in figure 1. A node $v_{i}$ maintains a table with two metrics $T_{i j}$ and $R_{i j}$, which contains an entry for each node $v_{j}$ to which $v_{i}$ has respectively transmitted packets to or received packets from. Node $v_{i}$ increments $T_{i j}$ on successful transmission of a packet to $v_{j}$ for $v_{j}$ to forward to another node, and increments $R_{i j}$ on successful receipt of a packet forwarded by $v_{j}$ that did not originate at $v_{j}$.

All nodes in the network continuously monitor their neighbors and update the list of those they have heard recently (Fig. 1.a). If the ID of an overheard node is not included in the table of overheard nodes a new entry is created. Otherwise, the existing entry is updated with a timestamp corresponding to the time the node was last overheard. Upon the creation of a new entry, a node schedules a task/event to check the behavior of the node whose ID has been saved in the new entry. Nodes randomly select a period of time between $\mathrm{T}_{\min }$ and $\mathrm{T}_{\max }$ to schedule the behavior checking task. This random selection seeks to reduce the possibility of two or more nodes starting a behavior check on the same node at the same time, wasting network bandwidth, battery energy and other network resources.

When a scheduled task is triggered in node $v_{k}$ to check $v_{j}$ 's behavior (Fig. 1.b), node $v_{k}$ broadcasts a metrics request packet $(M R E Q)$ with TTL $=1$ in the IP header. An MREQ includes the ID of the node emitting the request (SRC ID), the ID of the node whose behavior is to be checked (CHK_ID), an MREQ ID and a timestamp indicating the time at which the task was triggered. The MREQ ID is used in the same way as in some routing protocols which base their route discovery phase on broadcasting. If a node sees an MREQ that has the same MREQ ID and SRC_D of a packet seen before, the MREQ is dropped. This technique prevents flooding packets from traversing a zone of the network more than once. The tim estamp, on the other hand, is used to resolve conflicts when 
two nodes start a behavior check on the same node at almost the same time. In such cases, nodes can see which of the packets corresponds to the earlier triggered task and disregard the other. This does not require accurate synchronization of the nodes' clocks; approximate synchronization is all that need be assumed.

\section{a. OVERHEARING}

if node $\boldsymbol{v}_{\boldsymbol{k}}$ overhears a node $\boldsymbol{v}_{\boldsymbol{j}} \in U_{\boldsymbol{k}}$

- if node $\boldsymbol{v}_{\boldsymbol{j}}$ is not in $\boldsymbol{v}_{\boldsymbol{k}}$ 's table of overheard nodes

- . add node $\boldsymbol{v}_{\boldsymbol{j}}$ to $\boldsymbol{v}_{\boldsymbol{k}}$ 's table of overheard nodes

- . schedule an event to check $v_{j}$ 's behavior

- else

- update last time node $v_{\boldsymbol{j}}$ was heard

- endif

endif

\section{b. INITIATE BEHAVIOR CHECK}

if in node $\boldsymbol{v}_{\boldsymbol{k}}$ an event to check node $\boldsymbol{v}_{\boldsymbol{j}}$ 's behavior is triggered

- send a metrics request packet (MREQ) with node $v_{j}$ 's $I D$

- schedule another event to check $v_{j}$ 's behavior again at $t+T_{\max }$ endif

\section{c. REQUEST HANDLING}

if node $v_{k}$ receives a metrics request for node $\boldsymbol{v}_{\boldsymbol{j}}$

- if node $\boldsymbol{v}_{\boldsymbol{k}}$ has node $\boldsymbol{v}_{\boldsymbol{j}}$ in its table of overheard nodes

- rebroadcast metrics request packet (MREQ)

- . reschedule any event to check $\boldsymbol{v}_{\boldsymbol{j}}$ 's behavior

- . if node $\boldsymbol{v}_{\boldsymbol{k}}$ has metrics for node $\boldsymbol{v}_{\boldsymbol{j}}$

- . send a metrics reply (MREP) back to the requesting node

- endif

- else

- . ignore request

- endif

endif

\section{d. REPLY HANDLING}

if a request was sent out

- while there are more replies to be received for node $\boldsymbol{v}_{\boldsymbol{j}}$

- receive reply

- add received metrics to totals

- endwhile

- if $\sum_{\forall i \mid v_{i} \in U_{j}} R_{i j}<\left(1-\alpha_{\text {threshold }}\right) \sum_{\forall i \mid v_{i} \in U_{j}} T_{i j}$

$\cdot \cdot$

- node $\boldsymbol{v}_{\boldsymbol{j}}$ is misbehaving (detection)

- else

- node $v_{j}$ is not misbehaving (non-detection)

- endif

endif

Figure 1. Our algorithm pseudocode

The handling of requests (Fig. 1.c) is the heart of our limited broadcast algorithm. When a node receives an MREQ it first checks if the CHK ID is in its table of overheard nodes; if it is not the node ignores the MREQ and discards the check. However, if the CHK ID appears in its table then it rebroadcasts the MREQ with TTL $=1$ in the IP header. Setting the TTL to one allows our algorithm to control how far the broadcast of the MREQ is to go, instead of leaving this task to the IP protocol. Thus, every MREQ travels only one hop at a time, and is then analyzed and rebroadcast if the protocol so determines. By following this algorithm, our protocol is capable of tracking down nodes that have been in contact with the checked node, as illustrated in Figure 2. We assume transmissions can be overheard by vertically, horizontally and diagonally adjacent nodes. In the Figure, node $v_{7}$ is first in position $a$ where it can be overheard by nodes $v_{l}, v_{2}, v_{3}, v_{6}, v_{8}$, $v_{l l}, v_{l 2}$ and $v_{13}$. Each of these nodes makes an entry in their table of overheard nodes when $v_{7}$ first transmits and each of them schedules a task to check its behavior. At some point in time, $v_{7}$ decides to move following the path depicted in Figure 2 coming in contact with nodes $v_{14}, v_{17}, v_{18}, v_{19}, v_{20}, v_{23}, v_{24}$ and $v_{25}$. It finally stops in position $b$. In the Figure the scheduled behavior check initiation task (Fig. 1.b) in $v_{8}$ is the first to be triggered and the limited broadcast commences. All nodes that have overheard node $v_{7}$ re-broadcast the MREQ, whereas nodes such as $v_{4}, v_{9}$ and $v_{15}$ also receive the MREQ but ignore it because they have not overheard node $v_{7}$.

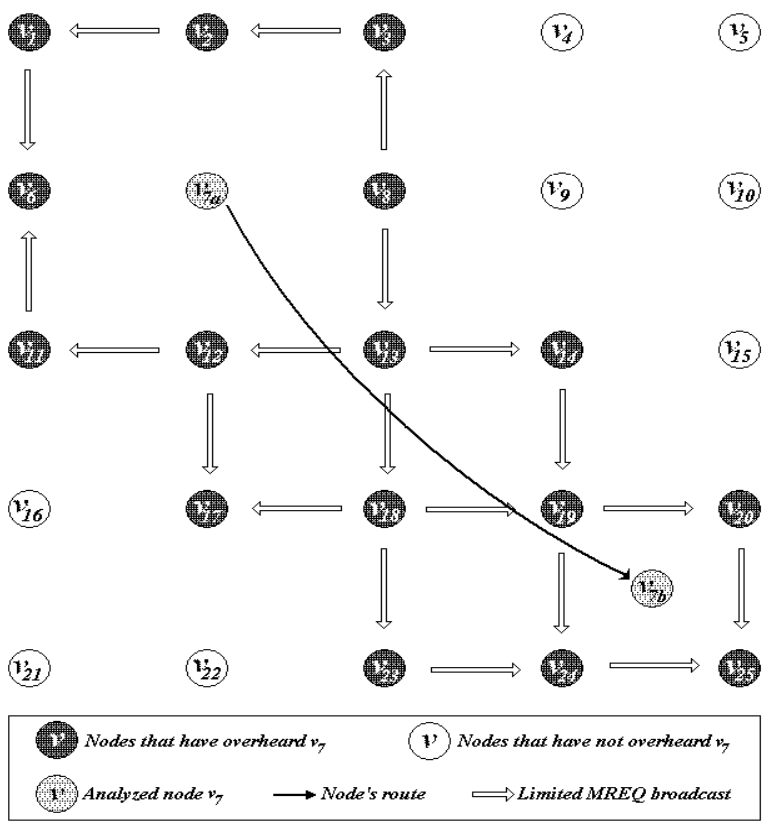

Figure 2. Example of limited broadcast to track down nodes that have overheard node $v_{7}$.

Once a node has decided whether to continue or not broadcasting a MREQ, it reschedules any pending task to check the behavior of the checked node specified in the CHK ID field of the MREQ. The new behavior checking task is scheduled in the same way as when a new entry is made in the table of overheard nodes, i.e. a period of time is randomly selected between $\mathrm{T}_{\min }$ and $\mathrm{T}_{\max }$. In this way if the random selection is uniformly distributed and the average frequency with which an active node's behavior is checked is:

avg freq $=\frac{1}{\left(T_{\min }+T_{\max }\right) / 2}=\frac{2}{\left(T_{\min }+T_{\max }\right)}$

The last step a node takes when it receives a MREQ is to check if it has any metrics $\left(\mathrm{R}_{\mathrm{ij}}\right.$ or $\left.\mathrm{T}_{\mathrm{ij}}\right)$ relating to the node being 
checked. If any of the metrics has a value other than zero the node returns a metrics reply packet (MREP) (Fig. 1.c) containing the metrics, but if the value of both metrics is zero then the node does not send back any response. In our scheme the task of finding a route back to the node that originated the MREQ has been left to the underlying routing protocol.

Reply handling is executed in the node that initiated the MREQ. This node, $v_{8}$ in Fig. 2, waits for a period of time in order to give all nodes with metrics about the checked node the opportunity of replying. When the time expires, the node checks the behavior of the analyzed node by verifying that equation (2) holds (Fig. 1.d). If it does not, it flags the checked node as a misbehaving one; this is a detection. Using a single detection to accuse a node is not sufficient since such an algorithm may lead to false accusations against correctly behaving nodes. A scheme in which multiple detections by different nodes are necessary to accuse a node is fairer to wellbehaved nodes, while keeping a high probability of correctly accusing misbehaving nodes. Thus, a system whose goal is to accuse misbehaving nodes, perhaps in order to punish them by withdrawing their right to transmit on the network, could use an approach similar to the distributed consensus mechanism proposed in SCAN [1].

\section{EVALUATION}

We perform our simulations using the GloMoSim simulation package. The results presented for each value are the average of 5 simulation runs. Our simulation parameters take the following values: i) The number of nodes in the network is 20, ii) Nodes are placed over an area of $200 \times 200$ squared meters, iii) Nodes move according to the random waypoint mobility model with a constant speed of $10 \mathrm{~ms}^{-1}$, iv) The pause time takes a value that is exponentially distributed with mean 30 seconds, v) the wireless transmission range of every node is 100 meters, vi) the link capacity is $2 \mathrm{Mbps}$, vii) the MAC layer protocol is the IEEE 802.11 DCF, viii) the underlying routing protocol is $\mathrm{AODV}$, and ix) the total simulation time for each scenario is 300 seconds. The network was set-up with $25 \%$ of nodes misbehaving by not forwarding all packets. Nodes check the behavior of active nodes within a period chosen uniformly between 15 and 30 seconds, and keep any overheard node in their tables for 60 seconds after the last time they are heard. The principal metric in our tests is the percentage of detections, which is assessed in terms of misbehavior threshold.

We consider the precision of our misbehavior detection algorithm in terms of the misbehavior threshold, which is the parameter $\alpha_{\text {threshold }}$ in equation (2), i.e. the maximum percentage of packets that a node is allowed to drop without being detected as a misbehaving node. In order to see properly the effect of the misbehavior threshold on nodes, simulations were carried out with nodes dropping packets with probabilities of $0 \%, 10 \%, 20 \%, 50 \%$ and $70 \%$ while the misbehavior threshold was varied from $0 \%$ to $100 \%$.

Figure 3 depicts the percentage of detections as a function of the misbehavior threshold for nodes exhibiting different probabilities of misbehavior. It can be inferred from the graph that the criterion to select an adequate misbehavior threshold depends on the level of trust required in the network. The lower the threshold is the more packets nodes need to forward to be considered well-behaved. However, since characteristics inherent to MANETs such as mobility and the noisy wireless medium can cause some packets to be lost (including packets of our own protocol), it also means that an increasing number of correctly behaving nodes can be falsely detected as misbehaving ones. Finally, it can also be seen from Fig. 3 that selecting a misbehavior threshold equal to a node's misbehaving probability prevents our approach from identifying misbehaving nodes with certainty, i.e. the probability of detection is approximately $50 \%$. These occurrences are all contained in the zone between $40 \%$ and $60 \%$ probability of detection in the figure.

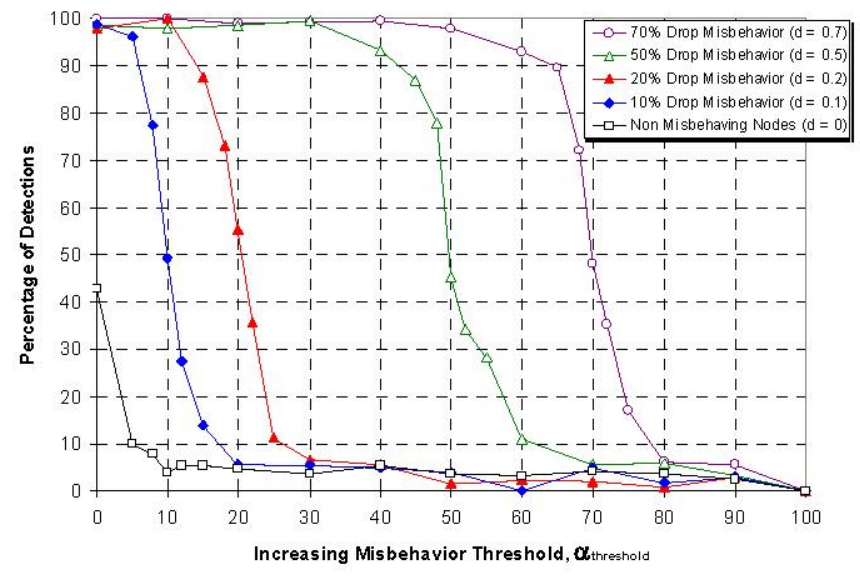

Figure 3. Percentage of positive detections as a function of the increasing misbehavior threshold (mean node speed $=10 \mathrm{~ms}^{-1}$ ).

\section{SUMMARY}

In this paper we have presented an algorithm that is capable of detecting packet forwarding misbehavior. The algorithm does not require high density networks in which many nodes can overhear each others' received and transmitted packets, but instead uses statistics accumulated by each node as it transmits to and receives data from its neighbors.

\section{REFERENCES}

[1] H. Yang, J. Shu, X. Meng, and S. Lu, "SCAN: Self-organized networklayer security in mobile ad hoc networks," IEEE Journal on Selected Areas in Communications, vol. 24, issue 2, pp. 261-273, February 2006.

[2] S. Marti, T. J. Giuli, K. Lai, and M. Baker, "Mitigating Routing Misbehavior in Mobile ad hoc networks," Proceedings of the $6^{\text {th }}$ ACM International Conference on Mobile Computing and Networking, pp. 255-265, August 2000

[3] K. A. Bradley, S. Cheung, N. Puketza, B. Mukherjee, and R. A. Olsson, "Detecting disruptive routers: a distributed network monitoring approach," Proceedings of the 1998 Symposium on Security and Privacy, pp. 115-124, May 1998.

[4] P. Karn, "MACA - a new channel access method for packet radio," ARRL/CRRL Amateur Radio $9^{\text {th }}$ Computer Networking Conference, pp 134-240, September 1990 .

[5] V. Bharghavan, A. Demers, S. Shenker, and L. Zhang, "MACAW: A Media Access Protocol for Wireless LANs," Proceedings of the ACM SIGCOMM Conference on Communications Architectures, Protocols and Applications, vol. 24, issue 4, pp. 212-225, 1994. 